\title{
Special Issue on Context-Awareness of Mobile Systems: Models, Algorithms and Applications
}

\author{
Phan Cong Vinh • Jun Suzuki
}

Published online: 26 January 2013

(C) Springer Science+Business Media New York 2013

\section{Foreword:}

This special issue, with papers contributed by prominent researchers from academia and industry, will serve as a reference material for researchers, scientists, professionals and students in computer science and computer engineering as well as developers and practitioners in computing and networking systems design by providing them with state-of-the-art research findings and future opportunities and trends. These contributions include some recent advances in contextawareness of mobile systems reflected in the six papers that we chose to invite for this special issue. In particular, the special issue covers various problems on models, algorithms and applications for context-awareness of mobile systems.

Paper 1 by Phan Cong Vinh and Nguyen Thanh Tung is both to give an in-depth analysis as well as to present the new material on the notion of context-aware computing, an idea that computing can both sense and react accordantly based on its environment. The paper formalizes context-awareness process using coalgebraic language, including the coalgebraic definition of context-awareness, bisimulation between context-awarenesses, homomorphism between contextawarenesses and context-awarenesses as coalgebras.

Paper 2 by Sara Montagna, Mirko Viroli, Jose Luis Fernandez Marquez, Giovanna Di Marzo Serugendo and Franco Zambonelli aims at deepening how self-organization can be injected in pervasive service ecosystems in terms of spatial structures and algorithms for supporting the design of contextaware applications. To this end, authors start from an existing

P. C. Vinh $(\bowtie)$

Department of Information Technology at NTT University,

Ho Chi Minh City, Vietnam

e-mail: pcvinh@ntt.edu.vn

J. Suzuki

Department of Computer Science at University of Massachusetts,

Boston, MA, USA

e-mail: jxs@cs.umb.edu classification of self-organization patterns, and systematically show how they can be supported in pervasive service ecosystems, and be composed to generate a self-organizing emergent behavior. A paradigmatic crowd steering case study is used to demonstrate the effectiveness of the approach.

Paper 3 by Julien Freudiger, Murtuza Jadliwala, JeanPierre Hubaux, Valtteri Niemi and Philip Ginzboorg discusses the ability to discover geographic proximity of specific communities of people (e.g., friends or neighbors). To do so, mobile devices must exchange some community identifiers or messages. Authors investigate privacy threats introduced by such communications, in particular, adversarial community detection. Authors use the general concept of community pseudonyms to abstract anonymous community identification mechanisms and define two distinct notions of community privacy using challenge-response methodology. An extensive cost analysis and simulation results throw further light on the feasibility of these mechanisms in the upcoming generation of wireless peer-to-peer networks.

Paper 4 by Andrei Olaru, Adina Magda Florea and Amal El Fallah Seghrouchni provides a model for a such middleware, based on software agents, in which context-awareness is implemented both in the agent's representation of context information, and in the logical topology of the agent system. The model is oriented towards decentralization of the system and relies mostly on local behavior. The paper also reports on several proof-of-concept applications that have been developed and tested using the proposed model, proving thus the validity of the approach.

Paper 5 by Antonio Coronato and Giuseppe De Pietro provides a new formal approach for the situation-awareness and the detection of abnormal behaviors of cognitive impaired people in situation-aware smart spaces. Instead of relying on the identification of deviations from normal behaviors, the approach is based on the specification and runtime verification of correctness properties. Situation Calculus is the formal method adopted to model the world; whereas, intelligent agents 
detect abnormal and dangerous situations. Dangerous situation recovery is also performed by ad-hoc intelligent agents.

Paper 6 by Nguyen Thanh Tung and Phan Cong Vinh provides the importance of considering neighboring nodes in the energy efficiency routing problem. After showing that the routing problem that considers the remaining energy of all sensor nodes is NP-complete, heuristics are proposed for the problem. Simulation results show that the routing algorithm that considers the remaining energy of all sensor nodes improves the system lifetime significantly compared to that of minimum transmission energy algorithms. Also, the energy dissipation of neighboring nodes accounts for a considerable amount of the total energy dissipation. Therefore, a method that reduces the energy dissipation by notifying the neighboring nodes to turn off their radio when not necessary is proposed. By reducing the unnecessary energy dissipation of the neighbors, the lifetime is increased significantly.

We owe our deepest gratitude to Dr. Nguyen Manh Hung Chairman and Rector of NTT University in Vietnam for his useful support, especially to all the authors for their valuable contribution to this special issue and their great efforts, and also to the referees for ensuring the high quality of the material presented here. All of them are extremely professional and cooperative. We wish to express our thanks to the Editor-inChief, Prof. Imrich Chlamtac, for his important assistance with the process of assembling the special issue.

\section{Phan Cong Vinh}

Jun Suzuki

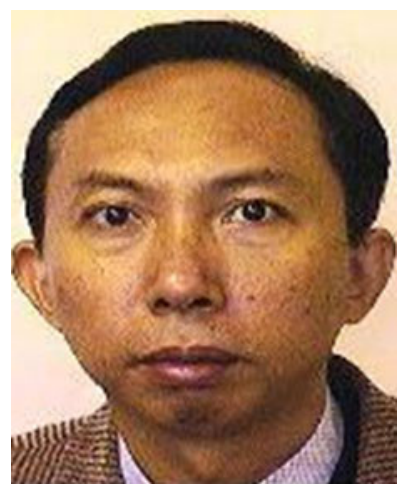

Phan Cong Vinh Received a $\mathrm{PhD}$ in computer science from London South Bank University (LSBU) in the United Kingdom, a BS in mathematics and an MS in computer science from Vietnam National University (VNU) in Ho Chi Minh City, and a BA in English from Hanoi University of Foreign Languages Studies in Vietnam. He finished his $\mathrm{PhD}$ dissertation with the title Formal Aspects of Dynamic Reconfigurability in Reconfigurable Computing Systems supervised by Prof. Jonathan P. Bowen at LSBU where he was affiliated with the Centre for Applied Formal Methods (CAFM) at the Institute for Computing Research (ICR). He joined research with Dr. Tomasz
Janowski at the International Institute for Software Technology (IIST) in Macao SAR, China, as a fellow in 2000. At present, he is a member of Nguyen Tat Thanh University (NTTU) to take on the responsibility of an IT Department's Head. He has been author or co-author of many refereed contributions published in prestigious journals, conference proceedings or edited books. He is the author of a book on computing science titled Dynamic Reconfigurability in Reconfigurable Computing Systems: Formal Aspects of Computing (2009); editor of two titles, Autonomic Networking-on-Chip: Bio-Inspired Specification, Development and Verification (CRC Press, 2012) and Formal and Practical Aspects of Autonomic Computing and Networking: Specification, Development and Verification (IGI Global, 2011); editor of two Special Issues, Context-Awareness of Mobile Systems: Models, Algorithms and Applications (Springer MONET (SCIE), 2012) and Advances in Autonomic Computing: Formal Engineering Methods for Nature-Inspired Computing Systems (Springer TCS, 2012). He has served on many conference program committees and has been general or technical (co)chair and (co)organizer of several international conferences such as ICCASA 2012, SCIS-ISIS 2012, ICCASA 2013 and iThings 2013. His research interests center on all aspects of formal methods, autonomic computing and networking, reconfigurable computing, ubiquitous computing, and applied categorical structures in computer science.

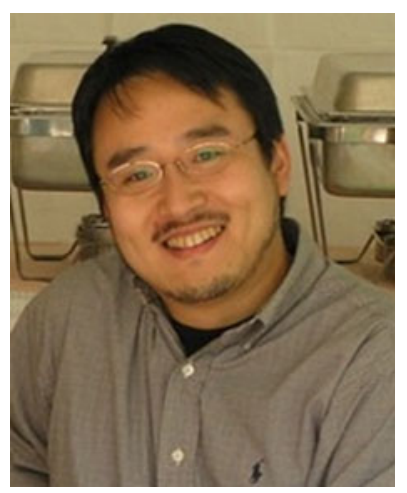

Junichi (Jun) Suzuki Is an Associate Professor of Computer Science at the University of Massachusetts, Boston. He received a Ph.D. in Computer Science from Keio University, Japan, in 2001. He was a postdoctoral research fellow at the University of California, Irvine (UCI) from 2001 to 2004 . Before joining UCI, he was with Object Management Group Japan, Inc., as Technical Director. His research interests include autonomous adaptive distributed systems, biologically-inspired computing, sustainable networking, molecular communication, model-driven software/performance engineering and multiobjective optimization. In these areas, he has authored three books, edited seven journal special issues, and published one industrial standard specification and $120+$ papers in international journals and conferences. He received nine best paper awards and two best poster awards at major conferences such as IEEE SPECTS 2008 and IEEE SCC 2007. He serves on the editorial boards for six international journals including Elsevier Nano Communication Networks Journal. $\mathrm{He}$ has chaired or co-chaired 15 international conferences such as BodyNets 2013, BIONETICS 2010 and ICSOC 2009. He has served on the steering committee of five conferences as well as the program committee of $120+$ conferences such as IEEE CEC, ACM GECCO, IEEE ICTAI, IEEE SECON, IEEE AINA, ACM/IEEE BIOSIGNALS and IEEE ICCCN. 